\title{
Устойчивые к полевым повреждениям структуры кремний-сверхтонкий окисел (42 нм)-поликремний
}

\author{
(С) Д.А. Белорусов, Е.И. Гольдман, В.Г. Нарышкина, Г.В. Чучева \\ Фрязинский фрилиал института радиотехники и электроники им. В.А. Котельникова \\ Российской академии наук, \\ 141190 Фрязино, Россия \\ 『 E-mail: gvc@ms.ire.rssi.ru
}

Поступила в Редакцию 24 августа 2020 г.

В окончательной редакции 2 сентября 2020 г.

Принята к публикации 2 сентября 2020 г.

\begin{abstract}
Приведены результаты исследований структур кремний-сверхтонкий окисел $(42 \AA)-$ поликремний, устойчивых к полевым повреждениям. Выяснилось, что происходящая с изменением полевого напряжения суммарная перезарядка сконцентрированных у границы раздела подложка-изолятор локализованных электронных состояний и неосновных носителей заряда близка к аналогичной характеристике структур с толщиной окисла $37 \AA$. Ток через $\mathrm{SiO}_{2}$ в состоянии обогащения полупроводника увеличивается с напряжением гораздо сильнее, чем в состоянии обеднения. Причем асимметрия вольт-амперных характеристик по отношению к полярности падающего на изоляторе напряжения у образцов с толщиной $\mathrm{SiO}_{2} 42 \AA$ более резко выражена, чем у структур с окислом $37 \AA$. Объяснение такой асимметрии возможно, если потенциальный рельеф в изоляторе имеет максимум, существенно смещенный к границе раздела окисел - поликремний, а потенциал на ветви со стороны полупроводника значительно спадает к контакту с подложкой.
\end{abstract}

Ключевые слова: структуры металл-диэлектрик-полупроводник, сверхтонкий окисел, полевое повреждение, высокочастотные вольт-фарадные характеристики, вольт-амперные характеристики.

DOI: 10.21883/FTP.2021.01.50379.9511

\section{1. Введение}

Сверхтонкие ( $<5$ нм) пленки окисла кремния - это основной тип изолирующих слоев современных и будущих устройств наномасштабного диапазона. Из-за малой толщины электрические поля, возникающие в таких слоях при управляющих воздействиях на активные элементы современных полупроводниковых устройств, достигают нескольких единиц $10^{6} \mathrm{~B} / \mathrm{cm}$. При этих значениях полей в $\mathrm{SiO}_{2}$ возникают значительные токи утечки и проявляются явления, ответственные за изменение свойств изолирующего промежутка - как обратимых, так называемое „повреждение окисла“, так и необратимых, мягкий пробой (soft breakdown). Подчеркнем, что, несмотря на допробойные значения указанных электрических полей, с ростом продолжительности воздействия вероятность перехода образца в необратимое состояние мягкого пробоя все равно возникает $[1,2]$. В нашей группе уже более десяти лет проводятся исследования изменений свойств сверхтонких окислов кремния после повреждения Si-МОП-структур в электрических полях [3-5]. Выяснилось [6], что имеется группа образцов с толщиной окисла $h=37 \AA$, у которых реакция на полевое воздействие типа повреждение практически отсутствует, т.е. с увеличением продолжительности выдержки при полевых напряжениях разной полярности вплоть до перехода в состояние мягкого пробоя у объектов практически не изменяются ни проводимость через окисел, ни распределение встроенного заряда. Естествен вопрос: насколько распространенным у сверхтонких окислов кремния явля- ется свойство „неповреждаемости“ при стрессах в электрических полях. Для его разрешения авторы обратились к объектам с другой толщиной окисла $-h=42 \AA$, но приготовленным по той же технологической схеме, как и в [6]. В качестве образцов использовались $\mathrm{Si-MOП-структуры} \mathrm{с} \mathrm{полевым} \mathrm{электродом} \mathrm{Al}-n^{+}-\mathrm{Si}: \mathrm{P}$ (концентрация доноров в поликремнии $N_{d}^{+} \approx 10^{20} \mathrm{~cm}^{-3}$, площадь полевого электрода $\left.S=1.6 \cdot 10^{-3} \mathrm{~cm}^{2}\right)$, изолированным от (100) n-Si-подложки слоем полученного при высокотемпературном окислении $\mathrm{SiO}_{2}$ с оптической толщиной $\sim 4.2$ нм.

\section{2. Эксперимент и результаты}

Структуры подвергались полевому стрессу при комнатной температуре при таких же полевых напряжениях $V_{g}$, как и при исследованиях повреждения сверхтонкого $\mathrm{SiO}_{2}[3,5]^{1}: V_{g}=-3.8 \mathrm{~B}$ (обеднение подложки), минимальное время выдержки 16 мин и $V_{g}=3.2$ В (обогащение подложки), минимальное время выдержки 20 мин. До и после стресса на автоматизированной установке проводились измерения вольт-амперной (BAX) и высокочастотных вольт-фарадных характеристик (ВФХ) объектов на частотах $1\left(C_{1}\right)$ и $0.5\left(C_{2}\right)$ МГц с использованием прецизионного измерителя LCR Agilent E4980A [4]. Peзультаты проведенных исследований приведены в данной работе.

\footnotetext{
${ }^{1}$ Уменьшение значения поля в окисле по сравнению с опытами [6] было не более чем на $13 \%$, что не существенно для целей исследования.
} 
Оказалось, что выбранные нами образцы, так же как и структуры с более тонким окислом [6], устойчивы к полевым воздействиям. Измеренные сразу же после стресса ВАХ и ВФХ были практически неотличимы от соответствующих характеристик, зафиксированных до выдержки объектов в электрическом поле: расхождения составляли не более $2 \%$ в отличие от структур [5], где после снятия повреждающего напряжения наблюдалось уменьшение емкостей в разы. В работе [6] у не повреждающихся структур была обнаружена существенная асимметрия BAX: при близких по модулю напряжениях токи в области сильного обеднения полупроводника были на порядки меньше, чем при обогащении кремния. Чтобы понять, присуще ли данное свойство объектам с толщиной окисла $42 \AA$, были проведены измерения ВАХ и ВФХ по аналогии с [6] в специальном режиме, когда значения высокочастотных емкостей и тока отвечают одному и тому же состоянию образца. Повторим суть схемы опыта. Для каждой точки измерений из положения полевое напряжение $V_{g}=0$ на образец подавалось заданное $V_{g}$; после окончания $\mathrm{RC}$ процессов $(<0.3 \mathrm{c})$ фиксировался ток через окисел $I$, затем в течение $3 \mathrm{c}$ через емкости $C_{1}$ и $C_{2}$. После этого поданное напряжение сбрасывалось до нуля, и структура выдерживалась 6 с. Общая продолжительность измерений тока и емкостей при одном и том же значении напряжения на полевом электроде $(6 \mathrm{c})$ существенно меньше, чем характерное время переходного процесса, связанного с перезарядкой локализованных электронных состояний на границе раздела (ГР) $\mathrm{Si}-\mathrm{SiO}_{2},(>100 \mathrm{c})$. Такая процедура позволяет при измерениях минимизировать длительность пребывания образца в стрессовых условиях. Данные ВФХ, соответствующие двум высоким частотам, дают возможность определять концентрацию доноров у ГР $\mathrm{Si}-\mathrm{SiO}_{2} N_{d}$, сопротивление полупроводниковой подложки $R_{b}$ и зависимости от полевого напряжения следующих величин: изгиба зон в полупроводнике $V_{s}$, падения внешнего напряжения на изолирующем слое $V_{i}$ и выраженной в $\mathrm{cm}^{-2}$ суммарной плотности встроенного заряда, заряда пограничных состояний и дырок на ГР $p_{\text {sq }}[4,5,7]$.

На рис. 1 представлены зависимости емкостей образца от напряжения, измеренные при комнатной температуре в состоянии до полевого стресса. Расчеты на основе данных ВФХ по методике, описанной в $[5,7]$, привели к значениям: $N_{d}=1.65 \cdot 10^{15} \mathrm{~cm}^{-3}$ и $R_{b}=73$ Ом. Эти величины почти такие же, как и полученные в [6]: $N_{d}=1.65 \cdot 10^{15} \mathrm{~cm}^{-3}$ и $R_{b}=88$ Ом. График $p_{\mathrm{sq}}\left(V_{g}\right)$ показан на рис. 2; состоянию плоских зон отвечает напряжение $V_{g}=-0.19$ В. Видно, что кривые, демонстрирующие перезарядку $\Gamma \mathrm{P} \mathrm{Si}-\mathrm{SiO}_{2}$, в объектах с толщиной окисла 4.2 нм (данная работа) и 3.7 нм [6] близки друг к другу: при изменении $V_{g}$ от -0.5 до $-6 \mathrm{~B}$ увеличение $p_{\mathrm{sq}}$ составило $\Delta p_{\mathrm{sq}}=1.0 \cdot 10^{13} \mathrm{~cm}^{-2}$ при $h=42 \AA$ и $\Delta p_{\mathrm{sq}}=1.4 \cdot 10^{13} \mathrm{~cm}^{-2}$ при $h=37 \AA$. Значения $\Delta p_{\mathrm{sq}}$ на порядок выше, чем в повреждающихся структурах $[5,8]$. Так же, как и [6] в области обеднения полупроводника,

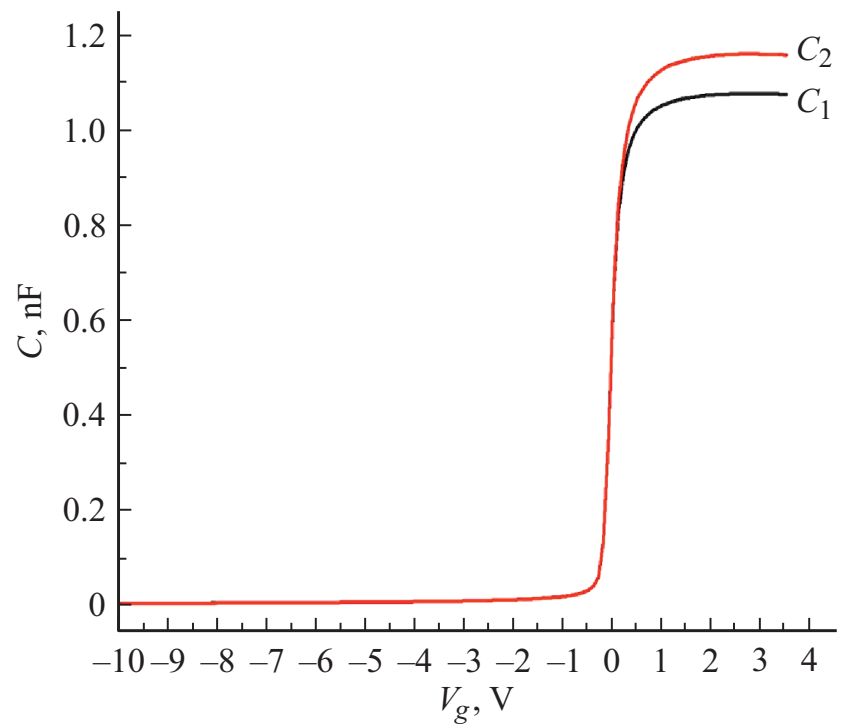

Рис. 1. Высокочастотные вольт-емкостные зависимости образцов, измеренные при комнатной температуре. Емкости: $C_{1}-$ при $1 \mathrm{MГц,} C_{2}-$ при 0.5 МГц.

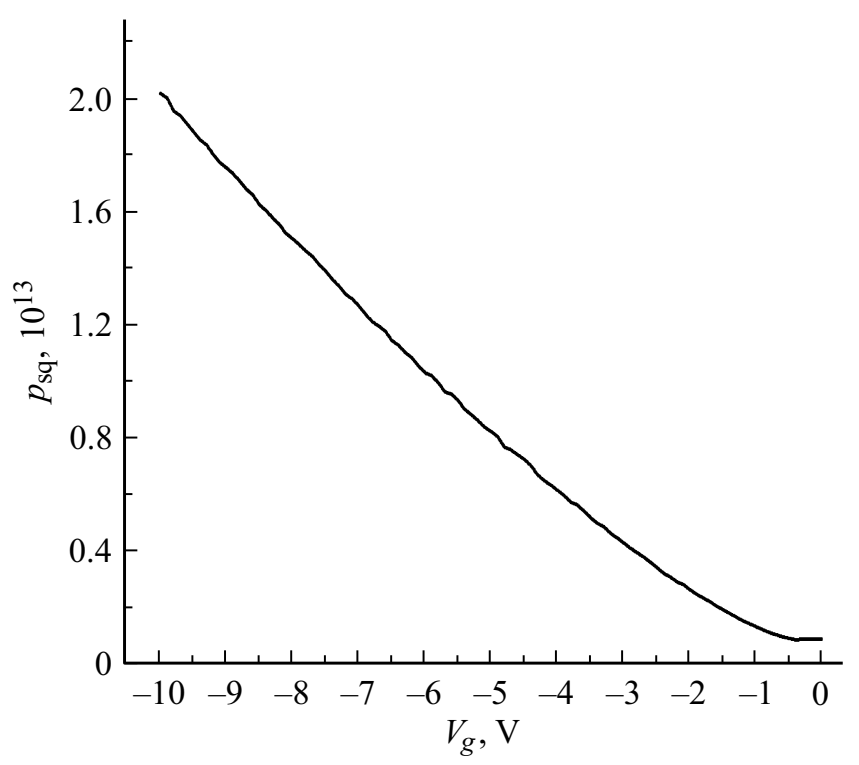

Рис. 2. Зависимость от полевого напряжения суммарной плотности встроенного заряда, заряда пограничных состояний и дырок на границе раздела $\mathrm{Si}-\mathrm{SiO}_{2}$.

оказалось, что $V_{i} \propto V_{g} / 2$ означает, что измерения проводились в нестационарном, неравновесном по отношению к неосновным носителям заряда (генерация дырок запаздывает) состоянии образца.

На рис. 3 показаны зависимости тока через окисел от падения напряжения на изолирующем слое при положительной (инжекция электронов из полупроводника) и отрицательной (инжекция электронов из полевого электрода) полярностях $V_{g}$. Для наглядности ВАХ приведены в линейном и в логарифмическом масштабах. Здесь $V=V_{g}$ при $V_{g}>0$ и $V=-V_{i}$ при $V_{g}<0$; гра- 


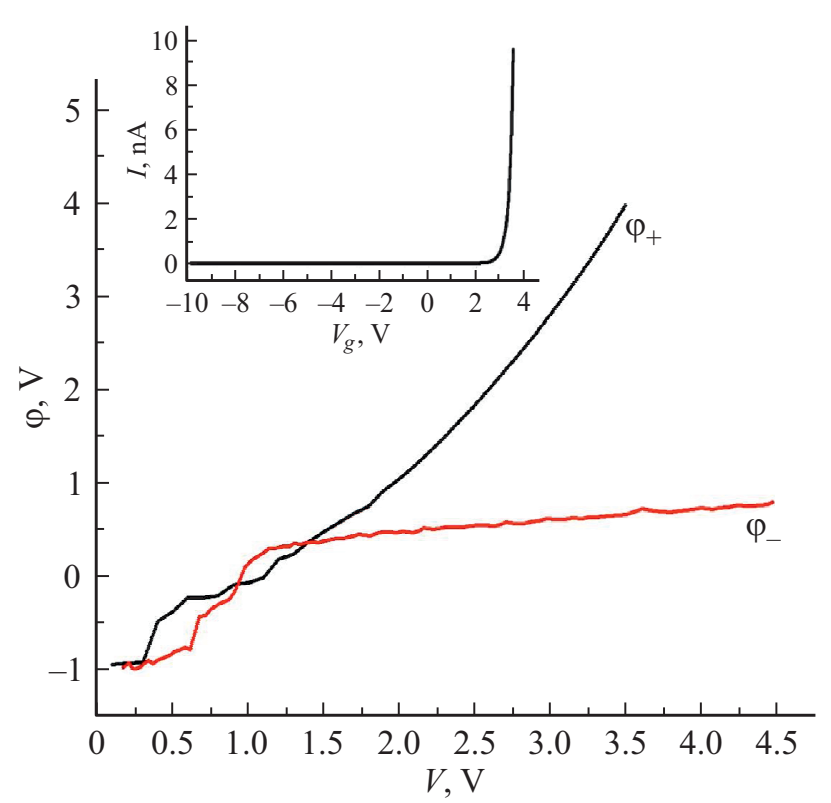

Рис. 3. Вольт-амперные характеристики изолирующего промежутка Si-MOП-структур, измеренные при комнатной температуре. $\varphi(V)$-- функция логарифма тока от напряжения на окисле. На вставке - зависимость тока от полевого напряжения в реальном масштабе.

фики ветвей тока в логарифмическом масштабе строились по правилу: $\varphi_{+}(V)=\lg \left[I\left(V_{g}\right) / I_{n}\right]-$ при $V_{g}>0$, $\varphi_{-}(V)=\lg \left[\left|I\left(V_{i}\right) / I_{n}\right|\right]$ при $V_{g}<0$, где $I_{n}=10^{-12} \mathrm{~A}-$ нормировочное значение тока. Отметим, что токи перезарядки $q S\left(d p_{s} / d t\right)(q-$ элементарный заряд, $t-$ время) на 1.5-2 порядка меньше $I$. Это следует из результатов измерений переходных характеристик тока и емкостей при нескольких напряжениях $V_{g}$. Из рис. 3 видно, что асимметрия ВАХ по отношению к полярности падающего на изоляторе напряжения у образцов с толщиной $\mathrm{SiO}_{2} 42 \AA$ более резко выражена, чем у структур с окислом $37 \AA ̊$. У объектов из настоящей работы ток в состоянии обогащения полупроводника в интервале напряжений $(0-3.5)$ В увеличивается на 5 порядков, а в состоянии обеднения всего на один. У образцов из работы [6] это соотношение было 5/3.

\section{3. Заключение}

Таким образом, можно констатировать, что, по крайней мере, для части сверхтонких слоев $\mathrm{SiO}_{2}$, полученных при высокотемпературном окислении кремния, типично свойство устойчивости к полевым повреждающим воздействиям. Выявление природы данного обстоятельства возможно только при постановке ряда специальных совместных технологических и физических исследований и не является целью данной работы. Обсудим особенности изолирующих слоев, лежащие в основе другого свойства подобных объектов - асимметричности ВАХ по отношению к полярности внешнего напряжения. Для сверхтонких пленок окиси кремния важным обстоятельством является не прямоугольная форма потенциального барьера, создаваемого изолирующим промежутком. Поскольку переходные слои между кристаллическим $\mathrm{Si}$ и $\mathrm{SiO}_{2}$, а также между окислом и полевым электродом из поли-Si занимают не менее $40 \%$ объема сверхтонкого диэлектрика [9], то они фактически во многом определяют свойства изолятора. Переходы от подложки к окислу и от окисла к поликремнию образуются в неодинаковых технологических процессах, имеют разную кристаллическую структуру. Поэтому естественно ожидать и разных (несимметричных) по форме координатных зависимостей потенциального изолирующего профиля на этих контактах. Для объяснения асимметричного вида ВАХ следует считать, что потенциальный рельеф в изоляторе имеет максимум, существенно смещенный к ГР окисел - поликремний; потенциал на ветви со стороны полупроводника значительно спадает к контакту с подложкой. При такой форме барьер „сьедается“ электрическим полем в $\mathrm{SiO}_{2}$ гораздо быстрее в случае инжекции электронов из полупроводника, чем из полевого электрода. Точный ответ на вопрос о форме потенциала в сверхтонком изолирующем промежутке должно дать построение реального рельефа, разделяющего подложку и полевой электрод, на основе методики, развитой в [10].

\section{Финансирование работы}

Работа выполнена в рамках государственного задания и частично поддержана Российским фондом фундаментальных исследований (проекты РФФИ № 18-29-11029-мк, № 19-07-00271-а и № 19-29-03042-мк).

\section{Конфликт интересов}

Авторы заявляют, что у них нет конфликта интересов.

\section{Список литературы}

[1] J.S. Suehle. In: Defects in Microelectronic Materials and Devices, ed. by D.M. Fleetwood, S.T. Pantelides, R.D. Schrimpf (Boca Raton, CRC Press, 2008) chap. 15, p. 437.

[2] J. Suñé, Ern. Y. Wu. In: Defects in Microelectronic Materials and Devices, ed. by D.M. Fleetwood, S.T. Pantelides, R.D. Schrimpf (Boca Raton, CRC Press, 2008) chap. 16, p. 465.

[3] Е.И. Гольдман, Н.Ф. Кухарская, В.Г. Нарышкина, Г.В. Чучева. ФТП, 45 (7), 974 (2011).

[4] Е.И. Гольдман, А.И. Левашова, С.А. Левашов, Г.В. Чучева. ФТП, 49 (4), 483 (2015).

[5] Е.И. Гольдман, С.А. Левашов, В.Г. Нарышкина, Г.В. Чучева. ФТП, 51 (9), 1185 (2017).

[6] Е.И. Гольдман, С.А. Левашов, Г.В. Чучева. ФТП, 53 (4), 481 (2019).

[7] Е.И. Гольдман, Н.Ф. Кухарская, С.А. Левашов, Г.В. Чучева. ФТП, 53 (1), 46 (2019). 
[8] Е.И. Гольдман, Ю.В. Гуляев, Г.В. Чучева. Радиотехника, 8, 58 (2015).

[9] А.П. Барабан, В.В. Булавинов, П.П. Коноров. Электроника слоев на кремнии (Л., Изд-во ЛГУ, 1988).

[10] Е.И. Гольдман, А.Г. Ждан, Н.Ф. Кухарская, М.В. Черняев. ФТП, 42 (1), 94 (2008).

Редактор Г.А. Оганесян

\section{Silicon-ultrathin oxide \\ $(4.2 \mathrm{~nm})$-polysilicon structures resistant to field damage}

\section{D.A. Belorusov, E.I. Goldman, V.G. Naryshkina,}

G.V. Chucheva

Fryazino Branch of the Kotelnikov Institute of Radioengineering and Electronics of Russian Academy of Sciences, 141190 Fryazino, Russia

Abstract Results of studies of silicon-silicon-ultrathin oxide $(42 \AA)$ - polysilicon structures structures stabile resistant to field damage are presented. It was found that the total recharging of localized electronic states and minority charge carriers, concentrated at the substrate-insulator interface, which occurs with a change in the field voltage and is close to the same characteristic of structures with an oxide thickness of $37 \AA$. The current, flowing through $\mathrm{SiO}_{2}$, in the enrichment state of the semiconductor increases with increasing voltage much more strongly than in the state of depletion. Moreover, the asymmetry of current-voltage characteristics in relation to the polarity of the voltage, falling on the insulator in samples with a thickness of $42 \AA \mathrm{SiO}_{2}$ is more pronounced than in structures with an oxide of $37 \AA$. An explanation for this asymmetry is possible, if the potential relief in the insulator has a maximum, significantly shifted to the oxide-polysilicon interface, and the potential on the branch from the semiconductor side significantly decreases to the contact with the substrate. 